ORIGINAL ARTICLE

\title{
Farinhas e subprodutos da laranja \\ sanguínea-de-mombuca: caracterização \\ química e aplicação em sorvete
}

\section{Flour and byproducts of mombuca blood orange: chemical characterization and application in ice cream}

\author{
Natalia Anilda de Souza Oliveira ${ }^{1}$, Dercy Olga Viana Winkelmann ${ }^{1}$, Thaise Maria Tobal ${ }^{1 *}$ (D) \\ ${ }^{1}$ Universidade Federal da Grande Dourados (UFGD), Faculdade de Ciências da Saúde, Dourados/MS - Brasil \\ *Corresponding Author: Thaise Maria Tobal, Universidade Federal da Grande Dourados (UFGD), Faculdade de \\ Ciências da Saúde, Rodovia Dourados-Itahum, km 12, Cidade Universitária, Caixa Postal: 533, CEP: 79804-970, \\ Dourados/MS - Brasil, e-mail: thaisetobal@ufgd.edu.br
}

Cite as: Oliveira, N. A. S., Winkelmann, D. O. V., \& Tobal, T. M. (2019). Flour and byproducts of mombuca blood orange: chemical characterization and application in ice cream. Brazilian Journal of Food Technology, 22, e2018246. https://doi.org/10.1590/1981-6723.24618

\begin{abstract}
Resumo
No presente estudo, foram caracterizados os componentes químicos de flavedo, albedo, semente, bagaço e das farinhas obtidas da laranja Citrus sinensis L. Osbeck sanguínea- de-mombuca e as farinhas foram utilizadas na substituição de gordura em sorvete. Foram desenvolvidas quatro formulações de sorvete de chocolate com diferentes concentraç̃̃es das farinhas obtidas, além da elaboração de uma formulação controle. Foram realizadas análises de composição centesimal e aceitabilidade sensorial, e os resultados foram avaliados por ANOVA e testes de médias de Tukey $(p \leq 0,05)$. $O$ teor de umidade de todas as farinhas obtidas encontra-se dentro dos limites estabelecidos na legislação (máximo de 15\%) e a quantidade de fibras indica que podem ser consideradas com alto conteúdo de fibras (maior que 6\%). Os resultados da avaliação sensorial indicaram que há maior aceitação da utilização da farinha do albedo na formulação de sorvetes do que da farinha de semente e flavedo de laranja. A utilização da farinha do albedo na substituição de gordura contribuiu para uma redução significativa de lipídeos $(33,3 \%)$ e aumento de fibras no sorvete $(59,2 \%)$. Portanto, considerando a composição química dos subprodutos da laranja sanguínea e das farinhas obtidas, estes têm um grande potencial para utilização em formulações de produtos alimentícios, assim como para extração de fibras.
\end{abstract}

Palavras-chave: Substituto de gordura; Fibra bruta; Composição centesimal; Aceitabilidade sensorial; Produtos alimentícios; Resíduos de laranja.

\begin{abstract}
In this study, the chemical components of flavedo, albedo, seed, bagasse and flours obtained from the orange Citrus sinensis L. Blood Osbeck of Mombuca were characterized, and the flours were used to replace fat in ice cream. Four chocolate ice cream formulations were developed, with different flour concentrations, besides the elaboration of a control formulation. Analysis of centesimal composition and sensory acceptability were carried out, and results were evaluated by ANOVA and Tukey mean test $(p \leq 0.05)$. The moisture content of all flours obtained is within the limits
\end{abstract}


established by the National Health Surveillance Agency, which determines a maximum of $15 \%$ moisture on flour and the amount of fibers is higher than that found in other studies with different fruits, what characterizes it as high fiber content (greater than 6\%). The results of sensory evaluation indicated that there is greater acceptance of the use of albedo flour in the formulation of ice cream than seed and Orange flavedo flour. The use of albedo flour as a fat substitute promoted significant reduction of lipids (33.3\%) and a fiber increase (59.2\%) in the ice cream. The fractions and flours obtained from blood orange have a great potential for use in food products formulations, given its favorable chemical composition, as well as for fiber extraction.

Keywords: Fat replacer; Crude fiber; Centesimal composition; Sensory analysis; Food products; Orange residues.

\section{Introdução}

A busca por alimentos mais saudáveis tem aumentado constantemente como alternativa para melhorar a qualidade de vida (Abreu et al., 2018) e, simultaneamente, a busca por inovação e desenvolvimento de produtos alimentícios que contribuam para atender às expectativas e necessidades dos consumidores também tem aumentado.

A fibra alimentar vem sendo estudada não só pelo seu grande potencial em prevenir as doenças crônicas não transmissíveis, mas também por suas propriedades na redução de gorduras em formulações (Barretto et al., 2015). Estudos mostram que a fibra contida na casca de frutas, como a laranja, tem um grande potencial na substituição de gordura na produção de alimentos, como os derivados da carne, fornecendo um produto de textura mais suave, muito semelhante ao convencional, reduzindo o teor de gordura e o valor energético (Talukder, 2015). Também pode ser usada na produção de pães isentos de gordura, com volume, sabor e textura aceitáveis (Stoll et al., 2015), assim como substituta de gordura na produção de sorvetes (Boff et al., 2013).

Atualmente, é muito significativa a produção de laranjas no Brasil (Instituto Brasileiro de Geografia e Estatística, 2018) e grande parte destas é processada em forma de suco e subprodutos cítricos, sendo o restante comercializado como frutas frescas. O albedo, parte branca e esponjosa localizada entre o flavedo e a polpa da laranja, envolve completamente o endocarpo, que é a porção comestível da fruta, protegendo-o de possíveis impactos (Ladaniya, 2008). O flavedo, constituído pela parte externa da casca, apresenta uma espessura fina e contém os carotenoides, pigmentos responsáveis pela coloração da fruta, além de óleos essências aromáticos que fornecem o aroma característico da laranja (Mendonça et al., 2006).

Essas partes, geralmente não utilizadas na fabricação do suco, contêm várias substâncias que podem ser aproveitadas como subprodutos na fabricação de ração animal e adubos utilizados na agropecuária (Nunes et al., 2009) e no enriquecimento de alimentos, além da indústria de cosméticos (Santos et al., 2010). As Citrus sinensis L. Osbeck (laranjas doces) são classificadas conforme a coloração de sua polpa. As laranjas claras apresentam a cor amarela da polpa e do suco, devido à presença de carotenoides, que variam entre $\mathrm{o}$ amarelo e o vermelho. Além dessa espécie, também há as que apresentam a coloração vermelha tanto da polpa como do suco, que são chamadas de laranjas sanguíneas (Paschoal et al., 2012).

As Citrus sinensis L. Osbeck sanguíneas-de-mombuca (laranjas sanguíneas) são originárias da região Mediterrânea, provavelmente de Malta ou da região da Sicília (Itália), e têm sido cultivadas há vários séculos, principalmente nos países Itália, Espanha, Marrocos, Argélia e Tunísia. São divididas nos grupos toroco, moro e sanguinello. Têm como características sua cor vermelha intensa, tanto da polpa como no suco, devido à presença de antocianinas, que apresentam atividade antioxidante (Hamedani et al., 2012).

No Brasil, ainda não apresentam interesse comercial, porém, devido ao seu potencial como alimento funcional, vêm sendo estudadas (Latado et al., 2008). A variedade encontrada no Brasil é da polpa vermelha ou laranja sanguíneo-falsa, que possuem a coloração vermelha somente na polpa, devido à presença de carotenoides, como o licopeno e maiores teores de betacaroteno (Saunt, 2000). Essa variedade, segundo 
Latado et al. (2008) apresenta de $275 \%$ a $675 \%$ mais licopeno do que a laranja pera. As principais variedades encontradas no Brasil são a laranja Bahia Cara-Cara, a sanguínea-de-mombuca e a Valência Puka.

Buscando atender aos consumidores, cada vez mais exigentes e preocupados com a saúde, a indústria de alimentos visa desenvolver produtos com quantidades reduzidas de açúcares e gorduras, sem que se interfira nas características organolépticas dos alimentos (Abreu et al., 2018).

No sorvete, a gordura contribui para uma textura suave, além de melhorar o corpo do produto e retardar o derretimento, auxiliando na estabilidade e reduzindo a necessidade de estabilizante (Pinheiro \& Penna, 2004). Os sorvetes que utilizam substituto de gordura na sua formulação têm a tendência de apresentar uma sensação de serem mais frios, ao degustá-los (Souza et al., 2010).

Considerando que resíduos cítricos possuem potencial para substituição de gordura, além de a laranja sanguínea não ser explorada comercialmente no Brasil e pela falta de trabalhos que caracterizem a composição desses resíduos, o presente trabalho objetivou caracterizar os componentes químicos de flavedo, albedo, semente, bagaço e das farinhas obtidas, além de utilizá-las na substituição de gordura em sorvete.

\section{Material e métodos}

As laranjas sanguíneas-de-mombuca (Citrus sinensis L. Osbeck) foram adquiridas de uma árvore de um pomar doméstico, localizada em Dourados-MS (Latitude: 22¹0'9.43”S; Longitude: 5449'12.09”O, sem tratos culturais sistemáticos, como poda, irrigação, controle de plantas invasoras e fertilização. Colhidas manualmente, em completa maturação de acordo com a coloração externa, foram acondicionadas em sacos plásticos e posteriormente conduzidas ao laboratório dentro de bolsas térmicas. Foram selecionadas, conforme sua forma e tamanho, e higienizadas, utilizando uma solução de hipoclorito de sódio a 50 ppm de cloro residual livre. Após a higienização, imediatamente os frutos foram cortados para separação de flavedo, albedo, semente e bagaço.

\subsection{Obtenção da farinha de flavedo, albedo, semente e bagaço da laranja sanguínea}

Para a elaboração da farinha, o albedo e o flavedo foram triturados com o auxílio de um liquidificador doméstico, e as sementes foram trituradas manualmente com o auxílio de um martelo de cozinha. Posteriormente, o albedo, o flavedo e as sementes, separadamente, foram lavados com água corrente, em sacos de algodão previamente esterilizados, com a finalidade de reduzir o sabor amargo presente nessas partes da laranja (Crizel et al., 2013).

As farinhas foram obtidas através de secagem em secador de bandejas a $75{ }^{\circ} \mathrm{C}$ até peso constante, totalizando aproximadamente 10 horas à velocidade de $1,0 \mathrm{~m} \cdot \mathrm{s}^{-1}$. Em seguida, foram trituradas novamente em liquidificador até obtenção da menor partícula possível e então peneiradas, utilizando-se peneiras com malhas de 20 mesh. Posteriormente, estas foram acondicionadas em sacos de polietileno selados e armazenados à temperatura ambiente, para posterior análise da composição centesimal. Para a produção dos sorvetes, foram utilizadas a farinha obtida do albedo e a mistura das farinhas obtidas do flavedo e da semente, na proporção de $73,5 \%$ e $26,5 \%$, respectivamente.

\subsection{Produção do sorvete}

Os ingredientes utilizados na preparação do sorvete foram adquiridos em comércio local. Foi elaborada uma formulação padrão, contendo $48 \%$ de gordura, e quatro formulações experimentais, contendo as farinhas obtidas, em diferentes concentrações, com base na quantidade de fibra utilizada na produção de sorvetes do estudo de Boff et al. (2013) e em testes prévios com relação às características sensoriais (Tabela 1). 
Tabela 1. Formulações do sorvete de chocolate padrão e dos sorvetes desenvolvidos com a adição das farinhas obtidas de flavedo, semente e albedo da laranja sanguínea.

\begin{tabular}{|c|c|c|c|c|c|}
\hline \multirow{2}{*}{ Ingredientes } & \multicolumn{5}{|c|}{ Composição (\%) } \\
\hline & $\mathbf{P a}^{\mathbf{a}}$ & $\mathbf{F 1}^{\mathbf{b}}$ & F2 $^{\mathbf{c}}$ & F3 $^{\mathrm{d}}$ & $\mathbf{F} 4^{\mathrm{e}}$ \\
\hline Leite desnatado & - & 73,3 & 72,9 & 73,1 & 73,1 \\
\hline Leite integral & 65,2 & - & - & - & - \\
\hline Leite em pó desnatado & & 0,7 & 0,7 & 0,7 & 0,7 \\
\hline Leite em pó integral & 5,1 & & & & \\
\hline Farinha da laranja & & 1,4 & 1,9 & 2,0 & 3,0 \\
\hline Creme de nata ( $48 \%$ de gordura) & 5,8 & & & & \\
\hline Açúcar & 11,6 & 11,0 & 10,9 & 11,0 & 11,0 \\
\hline Glicose & 4,2 & 1,1 & 1,1 & 1,1 & 1,1 \\
\hline Saborizante de chocolate & 4,9 & 5,1 & 5,1 & 4,8 & 4,8 \\
\hline Estabilizante & 3,2 & 7,3 & 7,3 & 7,3 & 7,3 \\
\hline
\end{tabular}

$\mathrm{P}=$ padrão. $\mathrm{F}=$ farinha. ${ }^{a}$ sorvete de chocolate padrão. ${ }^{\mathrm{b}}$ sorvete de chocolate com adição de $1,4 \%$ de farinha do flavedo e semente da laranja. ${ }^{\mathrm{c}}$ sorvete de chocolate com adição de $1,9 \%$ de farinha do flavedo e semente da laranja. ${ }^{\mathrm{d}}$ sorvete de chocolate com adição de $2,0 \%$ de farinha do albedo da laranja. ${ }^{e}$ sorvete de chocolate com adição de 3,0\% de farinha do albedo da laranja.

A primeira etapa da produção do sorvete consistiu na homogeneização dos ingredientes secos com o leite previamente aquecido a $40{ }^{\circ} \mathrm{C}$, até atingir a dissolução completa, e posteriormente na agitação em batedeira por 10 minutos, juntamente com o estabilizante. Em seguida, a massa obtida foi maturada em freezer durante quatro horas, homogeneizada por 10 minutos, armazenada no freezer por 48 horas, e decorrido esse período, novamente homogeneizada por mais 10 minutos, sendo então acondicionada em frascos de polietileno, sob congelamento até o momento da análise sensorial.

\subsection{Análise da composição centesimal e atividade de água}

Foi determinada a composição centesimal de flavedo, albedo e semente da laranja, bem como das farinhas obtidas de cada fração. O teor de umidade, proteínas, cinzas e lipídeos foi determinado segundo método da Association of Official Analytical Chemists (1990). A fibra alimentar foi determinada pelo método de Weende e os carboidratos totais obtidos por diferença. O fator de conversão de nitrogênio em proteínas utilizado foi de 6,38 e 6,25, para o sorvete e as farinhas, respectivamente (Cecchi, 2003).

A atividade de água das farinhas foi obtida por determinação direta, em equipamento AquaLab, em que a amostra foi acondicionada em cápsula, em equilíbrio com a atmosfera interna do equipamento.

\subsection{Avaliação sensorial}

A avaliação sensorial dos sorvetes foi realizada em um laboratório de análise sensorial, com 60 provadores não treinados, com idade entre 18 e 60 anos, entre estudantes e funcionários da Universidade, que consentiram em participar da pesquisa e assinaram um termo de consentimento livre e esclarecido, previamente aprovado pelo Comitê de ética em pesquisa com seres humanos (protocolo n. ${ }^{\circ}$ 1.005.028). As amostras foram servidas em copos codificados com números de três dígitos aleatórios, cerca de $30 \mathrm{~g}$ de cada amostra, em temperatura de $15{ }^{\circ} \mathrm{C}$, de forma monádica e aleatória, em cabines individuais sob luz branca. Para o teste de aceitação, foi utilizada uma escala hedônica estruturada de nove pontos, ancorada com os extremos "Desgostei muitíssimo" e "Gostei muitíssimo", avaliando os atributos cor, aroma, sabor, consistência e aceitação global (Meilgaard et al., 1999).

\subsection{Análise estatística}

Os resultados obtidos foram submetidos a uma Análise de Variância (ANOVA) e as médias, se diferentes, comparadas pelo teste de Tuckey ( $p \leq 0,05)$, utilizando o programa Origin 6.0. 


\section{Resultados e discussão}

\subsection{Composição centesimal}

Considerando os resultados obtidos da análise de composição centesimal das partes da laranja sanguínea (Tabela 2), existem diferenças significativas entre as frações da laranja para todos os constituintes analisados. A semente possui teor significativamente maior de proteínas do que as outras frações e o flavedo e o bagaço possuem quantidades similares de lipídeos e cinzas.

Tabela 2. Composição Centesimal da laranja sanguínea in natura.

\begin{tabular}{ccccccc}
\hline Fração & Umidade & Carboidrato & Proteína & Lipídios & Cinzas & Fibra bruta \\
\hline Flavedo & $84,2 \pm 0,3^{\mathrm{b}}$ & $11,9 \pm 0,3^{\mathrm{b}}$ & $1,5 \pm 0,8^{\mathrm{a}}$ & $1,8 \pm 0,1^{\mathrm{b}}$ & $0,62 \pm 0,07^{\mathrm{b}}$ & $3,39 \pm 0,01^{\mathrm{a}}$ \\
Albedo & $89,1 \pm 0,5^{\mathrm{a}}$ & $8 \pm 0,2^{\mathrm{d}}$ & $1,4 \pm 0,3^{\mathrm{a}}$ & $1,2 \pm 0,2^{\mathrm{c}}$ & $0,30 \pm 0,01^{\mathrm{c}}$ & $2,8 \pm 0,1^{\mathrm{b}}$ \\
Semente & $57,7 \pm 1,5^{\mathrm{c}}$ & $21,5 \pm 0,6^{\mathrm{a}}$ & $6,30 \pm 0,07^{\mathrm{b}}$ & $13,2 \pm 0,7^{\mathrm{a}}$ & $1,31 \pm 0,09^{\mathrm{a}}$ & $15,10 \pm 0,06^{\mathrm{c}}$ \\
Bagaço & $85,9 \pm 0,6^{\mathrm{b}}$ & $9,9 \pm 0,2^{\mathrm{c}}$ & $1,6 \pm 0,2^{\mathrm{a}}$ & $2,05 \pm 0,06^{\mathrm{b}}$ & $0,51 \pm 0,05^{\mathrm{b}}$ & $1,04 \pm 0,02^{\mathrm{d}}$ \\
\hline
\end{tabular}

Letras diferentes na mesma coluna indicam diferença significativa pelo teste de Tukey $(p \leq 0,05)$.

Dos subprodutos analisados, o que apresentou umidade significativamente maior foi o albedo, sendo similar aos valores encontrados na laranja pera $(89,7 \%)$, na tangerina $(89,2 \%)$ e no limão tahit $(87,4 \%)$ (Universidade Estadual de Campinas, 2011), e a menor umidade foi encontrada na semente, semelhante ao valor da semente da laranja pera, de 55,4\% (Kobori \& Jorge, 2005). A semente apresenta uma quantidade de lipídeos muito maior do que as demais partes, sendo similar ao resultado encontrado por Kobori \& Jorge (2005), que quantificou 15\% de lipídeos na semente da laranja pera e 12,3\% na semente de goiaba.

Quanto ao teor de carboidratos, todas as partes diferiram significativamente entre si, sendo que o substrato que possui maior e menor teor de carboidratos é a semente e o albedo, respectivamente. A maior quantidade de proteína e cinzas foi encontrada na semente, sendo que as demais partes não apresentaram diferença significativa entre si, exceto pelo flavedo com relação às cinzas.

A quantidade de fibras encontrada foi diferente entre todas as amostras, sendo que a semente possui o teor mais elevado, seguida do flavedo, albedo e bagaço. A semente apresenta maior quantidade de todos os componentes analisados neste estudo, justamente pelo fato de apresentar o menor teor de umidade, possuindo maior concentração de sólidos solúveis.

Com relação às farinhas obtidas das partes da laranja sanguínea, o rendimento foi de $36 \%, 25 \%, 18 \% \mathrm{e}$ $9 \%$, para flavedo, albedo, semente e bagaço, respectivamente, e o teor de umidade de todas (Tabela 3 ) encontra-se dentro dos limites estabelecidos pela Agencia Nacional de Vigilância Sanitária, que estipula o máximo de 15\% (Brasil, 2005).

Tabela 3. Composição Centesimal das farinhas obtidas das partes da laranja sanguínea.

\begin{tabular}{ccccccc}
\hline Fração & Umidade & Carboidrato & Proteína & Lipídios & Cinzas & Fibra Bruta \\
\hline Flavedo & $8,7 \pm 0,9^{\mathrm{a}}$ & $73,1 \pm 0,5^{\mathrm{c}}$ & $6,3 \pm 0,2^{\mathrm{b}}$ & $8,0 \pm 0,4^{\mathrm{b}}$ & $3,9 \pm 0,3^{\mathrm{a}}$ & $21,0 \pm 0,5^{\mathrm{c}}$ \\
Albedo & $7,8 \pm 0,1^{\mathrm{a}}$ & $77,5 \pm 0,3^{\mathrm{b}}$ & $4,1 \pm 0,6^{\mathrm{c}}$ & $7,2 \pm 0,4^{\mathrm{b}}$ & $3,5 \pm 0,1^{\mathrm{b}}$ & $26,9 \pm 0,6^{\mathrm{b}}$ \\
Semente & $6,0 \pm 0,8^{\mathrm{b}}$ & $41,2 \pm 0,4^{\mathrm{d}}$ & $15,3 \pm 0,4^{\mathrm{a}}$ & $35,0 \pm 0,2^{\mathrm{a}}$ & $2,6 \pm 0,2^{\mathrm{c}}$ & $35,0 \pm 1,1^{\mathrm{a}}$ \\
Bagaço & $7,2 \pm 2,3^{\mathrm{ab}}$ & $79,9 \pm 1,0^{\mathrm{a}}$ & $4,7 \pm 0,1^{\mathrm{c}}$ & $5,1 \pm 0,8^{\mathrm{c}}$ & $3,2 \pm 0,9^{\mathrm{b}}$ & $6,4 \pm 0,4^{\mathrm{d}}$ \\
\hline
\end{tabular}

Letras diferentes na mesma coluna indicam diferença significativa pelo teste de Tukey $(p \leq 0,05)$.

O teor de lipídeos e proteínas da farinha obtida da semente é similar ao encontrado no estudo de Rosa (2013), com 35,9\% e 15,3\% de lipídeos e proteínas, respectivamente, e na laranja da variedade pera-rio. Reda et al. (2005) também encontraram valores próximos de lipídeos para a farinha dos limões rosa e siciliano, com $32 \%$ e $38,3 \%$, respectivamente. 
Com relação à farinha do albedo da laranja, considerando o estudo de Bublits et al. (2013), que analisou a farinha da laranja tipo valência, com exceção de umidade e proteína, todos os componentes foram encontrados em maior concentração do que neste estudo, que quantificou $11,8 \%, 5,9 \%, 63,4 \%, 0,4 \%, 2,5 \%$ e 16,2\% de umidade, carboidratos, lipídios, cinzas e fibras, respectivamente. Já no estudo de Santos et al. (2010), que analisou a farinha da laranja pera, os valores obtidos foram similares, 7,8\%, 3,8\%, 3,7\% e 46,1\% de umidade, cinzas, proteínas e carboidratos, respectivamente.

A maior concentração de minerais foi encontrada na farinha do flavedo, seguida das de albedo e bagaço, sendo os valores encontrados similares ao de outro estudo realizado com a laranja pera (Santos et al., 2010). Quanto à quantidade de fibras, todas as amostras diferiram significativamente entre si, sendo os valores encontrados superiores às quantidades encontradas em outros estudos com frutas, como laranja pera e maracujá (Lima et al., 2012; Santos et al., 2010), destacando o potencial das farinhas obtidas da laranja sanguínea para obtenção e aplicação de fibras.

\subsection{Análise sensorial}

Dos atributos avaliados sensorialmente nas formulações de sorvete, a cor e o aroma não diferiram significativamente entre as amostras (Tabela 4), exceto pela formulação F1, que apresentou nota inferior no aspecto aroma. Todas as outras receberam nota acima de 7 , que corresponde a expressão "gostei moderadamente". Entretanto, para os atributos aparência, sabor, textura e aceitação global, a amostra padrão recebeu nota significativamente maior, com exceção da amostra F4, que apresentou nota similar à da amostra padrão, no atributo aparência.

Tabela 4. Aceitabilidade sensorial dos atributos avaliados das formulações dos sorvetes de chocolate.

\begin{tabular}{|c|c|c|c|c|c|}
\hline \multirow{2}{*}{ Atributos } & \multicolumn{5}{|c|}{ Formulações } \\
\hline & Padrão $^{1}$ & $\mathrm{F1}^{2}$ & F2 $^{3}$ & F3 $^{4}$ & $F 4^{5}$ \\
\hline Aparência & $7,4 \pm 1,4^{\mathrm{a}}$ & $6,7 \pm 2,0^{b}$ & $6,6 \pm 1,9^{b}$ & $6,4 \pm 1,6^{b}$ & $6,9 \pm 1,7^{\mathrm{ab}}$ \\
\hline Cor & $7,4 \pm 1,6^{a}$ & $7,4 \pm 1,5^{a}$ & $7,5 \pm 1,5^{\mathrm{a}}$ & $7,0 \pm 1,7^{\mathrm{a}}$ & $7,4 \pm 1,3^{\mathrm{a}}$ \\
\hline Aroma & $7,6 \pm 1,7^{\mathrm{a}}$ & $6,6 \pm 1,2^{b}$ & $7,0 \pm 1,9^{\mathrm{ab}}$ & $7,1 \pm 1,7^{\mathrm{ab}}$ & $7,3 \pm 1,4^{a}$ \\
\hline Sabor & $7,6 \pm 1,6^{\mathrm{a}}$ & $4,3 \pm 2,2^{b}$ & $3,3 \pm 1,7^{\mathrm{c}}$ & $5,6 \pm 1,9^{d}$ & $5,9 \pm 1,9^{d}$ \\
\hline Textura & $6,6 \pm 2,2^{\mathrm{a}}$ & $5,2 \pm 2,1^{b}$ & $5,2 \pm 2,1^{b}$ & $5,1 \pm 2,0^{\mathrm{b}}$ & $5,6 \pm 2,2^{b}$ \\
\hline Aceitação global & $7,3 \pm 1,6^{\mathrm{a}}$ & $5,1 \pm 2,3^{b}$ & $4,4 \pm 2,0^{b}$ & $5,8 \pm 1,8^{c}$ & $6,0 \pm 1,8^{c}$ \\
\hline
\end{tabular}

${ }^{1}$ sorvete padrão, com gordura. ${ }^{2} \mathrm{e}^{3}$ sorvete com $1,4 \%$ e $1,9 \%$, respectivamente, de farinha do flavedo e semente da laranja; ${ }^{4} \mathrm{e}^{5}$ sorvete com $2,0 \%$ e $3,0 \%$, respectivamente, de farinha do albedo. Letras diferentes na mesma linha indicam diferença significativa de acordo com o teste de Tukey $(p \leq 0,05)$.

Com relação ao sabor e à aceitação global, as formulações F3 e F4 foram as que receberam maior nota em seguida à formulação padrão, sendo que a $\mathrm{F} 4$ foi a que obteve a melhor média, correspondendo à expressão "gostei ligeiramente" do teste de aceitabilidade aplicado. Isso indica que o tipo de fração da laranja adicionada ao sorvete foi determinante na avaliação desses atributos, sendo mais aceitável o uso da farinha obtida do albedo da laranja.

Nos estudos realizados por Boff et al. (2013), que utilizaram a fibra da laranja amarga na elaboração de sorvete, e por Crizel et al. (2013), que elaboraram sorvete de chocolate com a farinha do flavedo, semente e albedo da laranja pera, foram avaliados os atributos cor, odor e textura, que não apresentaram diferença significativa em relação ao sorvete controle. Todas as amostras receberam notas acima de 7, equivalente na escala hedônica utilizada à expressão "gostei moderadamente".

Dervisoglu \& Yazici (2006) desenvolveram sorvete com a adição da farinha de frutas cítricas como substituta de gordura e observaram que, quando adicionada uma quantidade maior de emulsificante, ocorreu uma maior aceitação do sorvete. Possivelmente, as notas baixas para o sabor do sorvete adicionado das farinhas, sem o emulsificante, ocorreram por resquícios de amargor da fruta, pelo fato de a farinha apresentar substâncias, como óleos essenciais e naringina (Boff et al., 2013). No presente estudo, além de não ter sido 
utilizado estabilizante nas formulações, os sorvetes foram elaborados de forma artesanal, o que afeta tanto o sabor como a textura, e, consequentemente, a impressão global.

Considerando os resultados da avaliação sensorial, foi realizada a caracterização química das amostras que obtiveram melhor aceitação: padrão e formulação F4 (Tabela 5), considerando que apesar de a formulação $\mathrm{F} 3$ não diferir significativamente da F4 na aceitação global, a F4 possui aspectos mais interessantes quanto à composição, já que foi adicionada de uma maior quantidade da farinha do albedo.

Tabela 5. Composição centesimal dos sorvetes de chocolate controle e da formulação com adição de farinha do albedo da laranja (F4*), que obteve melhor aceitação sensorial.

\begin{tabular}{ccccccc}
\hline Sorvete & Umidade & Carboidrato & Proteína & Lipídios & Cinzas & Fibra Bruta \\
\hline Controle & $58,9 \pm 0,8^{\mathrm{a}}$ & $33,1 \pm 0,4^{\mathrm{a}}$ & $4,7 \pm 0,1^{\mathrm{a}}$ & $1,5 \pm 0,1^{\mathrm{a}}$ & $0,92 \pm 0,02^{\mathrm{a}}$ & $0,98 \pm 0,07^{\mathrm{a}}$ \\
F4 & $60,8 \pm 0,8^{\mathrm{a}}$ & $31,6 \pm 0,4^{\mathrm{a}}$ & $4,7 \pm 0,8^{\mathrm{a}}$ & $0,5 \pm 0,1^{\mathrm{b}}$ & $0,92 \pm 0,04^{\mathrm{a}}$ & $1,56 \pm 0,05^{\mathrm{b}}$ \\
\hline
\end{tabular}

F4* = sorvete de chocolate com adição de $2,96 \%$ de farinha do albedo da laranja. Letras diferentes na mesma coluna indicam diferença significativa de acordo com o teste de Tukey $(p \leq 0,05)$.

Não houve diferença significativa entre as amostras de sorvete controle e F4 com relação a umidade, carboidratos, proteínas e cinzas, indicando que a adição da farinha na formulação não alterou esses componentes. Porém, foram expressivos a redução do teor de lipídeos e o acréscimo de fibras na formulação F4, quando comparada ao sorvete convencional. O lipídio, encontrado em maior quantidade nos sorvetes convencionais, garante maior maciez, cremosidade, durabilidade e redução da sensação de frio (Lamounier et al., 2012). O teor de fibras encontrado na formulação F4 é similar ao determinado em sorvetes de frutas do cerrado (Morzelle et al., 2012), sendo importante para a integridade estrutural, o volume e a capacidade de homogeneização do produto (Monteiro et al., 2006).

\section{Conclusões}

As farinhas obtidas de flavedo, albedo, semente e bagaço da laranja sanguínea apresentaram umidade dentro dos limites estabelecidos pela legislação vigente e elevado teor de fibras.

As frações e as farinhas obtidas da laranja sanguínea têm um grande potencial para utilização em formulações de produtos alimentícios, dada sua composição química, assim como podem ser usadas para a extração de fibras.

O sorvete de chocolate desenvolvido obteve uma aceitabilidade sensorial razoável, sendo mais aceitável a formulação desenvolvida com farinha do albedo da laranja, que apresentou redução significativa de lipídeos e acréscimo de fibras, quando comparada com a formulação padrão.

\section{Referências}

Abreu, E., Preci, D., Zeni, J., Steffens, C., \& Steffens, J. (2018). Desenvolvimento de Frozen Yogurt de iogurte em pó de leite de ovelha. Revista Ceres, 65(1), 7-15. http://dx.doi.org/10.1590/0034-737x201865010002

Association of Official Analytical Chemists - AOAC. (1990). Official methods of analysis of the Association of Official Analytical Chemists (15th ed., 2 Vol.). Gaithersburg: AOAC.

Barretto, A. C. S., Pacheco, M. T. B., \& Pollonio, M. A. R. (2015). Effect of the addition of wheat fiber and partial pork back fat on the chemical composition, texture and sensory property of low-fat bologna sausage containing inulin and oat fiber. Food Science and Technology, 35(1), 100-107. http://dx.doi.org/10.1590/1678-457X.6496

Boff, C. C., Crizel, T. M., Araujo, R. R., Rios, A. O., \& Flôres, S. H. (2013). Desenvolvimento de sorvete de chocolate utilizando fibra de casca de laranja como substituto de gordura. Ciência Rural, 43(10), 1892-1897. http://dx.doi.org/10.1590/S0103-84782013001000026

Brasil. Comissão Nacional de Normas e Padrões para Alimentos. (2005, setembro 22). Resolução RDC n 263 , de 22 de setembro de 2005. Aprovam regulamentos técnicos para produtos de cereais, amidos, farinhas e farelos. Diário Oficial [da] República Federativa do Brasil, Brasília. Recuperado em 7 de fevereiro de 2018, de http://e-legis.anvisa.gov.br/leisref/public/showAct.php?id=18822\&word=

Bublits, S., Emmanoulidis, P., Oliveira, R. S. M., Rohlfes, B. L. A., Baccar, M. N., Corbellin, A. V., \& Marquardt, L. (2013). Produção de uma farinha de albedo de laranja como forma de aproveitamento de resíduo. Revista Jovens Pesquisadores, 3(2), 112-121. 
Cecchi, H. M. (2003). Fundamentos teóricos e práticos em análise de alimentos (2. ed.). Campinas: UNICAMP. http://dx.doi.org/10.7476/9788526814721.

Crizel, T. M., Jablonski, A., Rios, A. O., Rech, R., \& Flores, S. H. (2013). Dietary fiber from orange byproducts as a potential fat replacer. Food Science and Technology, 53(1), 9-14.

Dervisoglu, M., \& Yazici, F. (2006). Effect of citrus fibre on the physical, chemical and sensory properties of ice cream. Food Science \& Technology International, 12(2), 159-164. http://dx.doi.org/10.1177/1082013206064005

Hamedani, M., Rabiel, V., Moradi, H., Ghanbari, A., \& Azimi, R. M. (2012). Determination of storage duration and temperature effects on fruit quality parameters of blood orange (Citrus sinensis CV. Torocco). Biharean Biologist, 1, 10-13.

Instituto Brasileiro de Geografia e Estatística - IBGE. Grupo de Coordenação de Estatísticas Agropecuárias - GCEA. Diretoria de Pesquisas, Coordenação de Agropecuária, Levantamento Sistemático da Produção Agrícola. (2018). Estatística da produção agrícola. Rio de Janeiro: IBGE.

Kobori, C. N., \& Jorge, N. (2005). Caracterização dos óleos de algumas sementes de frutas como aproveitamento de resíduos industrias. Ciência e Agrotecnologia, 29(5), 1008-1014. http://dx.doi.org/10.1590/S1413-70542005000500014

Ladaniya, M. S. (2008). Citrus fruit: Biology, technology and evaluation. San Diego: Elsevier.

Lamounier, M. L., Araújo, R. A. B. M., Lamounier, M. L., \& Morzelle, M. C. (2012). Desenvolvimento de sorvete enriquecido com fibras de linhaça e lactobacilos vivos e sua viabilidade. Revista do Instituto de Laticínios Cândido Tostes, 67(387), 57-63. http://dx.doi.org/10.5935/2238-6416.20120050

Latado, R. R., Tognato, P. C., Silva-Stenico, M. E., Nascimento, L. M., \& Santos, P. C. (2008). Acúmulo de antocianinas e características físicas e químicas de frutos de laranjas sanguíneas durante o armazenamento a frio. Revista Brasileira de Fruticultura, 30(3), 604-610. http://dx.doi.org/10.1590/S0100-29452008000300007

Lima, E. S., Schwertz, M. C., Sobreira, C. R. C., \& Borras, M. R. L. (2012). Efeito hipoglicemiante da farinha do fruto de maracujá-domato (Passiflora nítida Kunth) em ratos normais e diabéticos. Revista Brasileira de Plantas Medicinais, 14(2), 383-388. http://dx.doi.org/10.1590/S1516-05722012000200020

Meilgaard, M., Civille, G. V., \& Carr, B. T. (1999). Sensory evaluation techniques (3rd ed.). Boca Raton: CRC. http://dx.doi.org/10.1201/9781439832271.

Mendonça, L. M. V. L., Conceição, A., Piedade, J., Carvalho, V. D., \& Theodoro, V. C. A. (2006). Caracterização da composição química e do rendimento dos resíduos industriais do limão Tahiti (Citrus latifolia Tanaka). Food Science and Technology, $26(4), 5$. http://dx.doi.org/10.1590/S0101-20612006000400025

Monteiro, S. C., Carpes, S. T., Kalluf, V. H., Dyminski, D. S., \& Cândido, L. M. B. (2006). Evolução dos substitutos de gordura utilizados na Tecnologia de alimentos. Boletim do Centro de Pesquisa e Processamento de Alimentos, 24(2), 347-362. http://dx.doi.org/10.5380/cep.v24i2.7494

Morzelle, C. M., Lamouunier, L. M., Souza, C. E., Salgado, M. J., \& Vilas-Boas, B. V. E. (2012). Características físico-química e sensorial de sorvetes à base de frutos do cerrado. Revista do Instituto de Laticínios Cândido Tostes, 67(387), 70-78. http://dx.doi.org/10.5935/2238-6416.20120052

Nunes, P. M. P., Smolarek, F. S. F., Kaminki, G. A. T., Fin, M. T., Zanin, S. M. W., \& Miguel, O. L. G. (2009). A importância do aproveitamento dos resíduos industrias da semente de Citrus. Revista Visão Acadêmica, 10(1), 97-110.

Paschoal, D., Pinheiro, T. T., Figueira, A., \& Latado, R. R. (2012). Transformação de laranja doce visando a superexpressão do gene $\beta$-licopeno syntase (Iniciação científica). Campinas: Instituto Agronômico de Campinas.

Pinheiro, S. V. M., \& Penna, B. L. A. (2004). Substitutos de Gordura: tipos e aplicações em produtos lácteos. Alimentos e Nutrição, 15(2), 175-186.

Reda, S. Y., Leal, E. S., Batista, E. A. C., Barana, A. C., Schnitzel, E., \& Carneiro, P. I. B. (2005). Caracterização dos óleos das sementes de limão rosa e limão siciliano em resíduo agroindustrial. Food Science and Technology, 25(4), 672-676. http://dx.doi.org/10.1590/S0101-20612005000400008

Rosa, P. D. (2013). Resíduo de laranja: Estudo da cinética de secagem das sementes e influência da temperatura na qualidade do óleo (Dissertação de mestrado). Instituto de Biociências, Letras e Ciências Exatas, Universidade Estadual Paulista "Julio de Mesquita Filho", São José do Rio Preto.

Santos, A. J. A. O., Silva, I. C. V., Leite, M. L. C., Soares, M. S., \& Marcellini, P. S. (2010). Desenvolvimento de biscoito de chocolate a partir da incorporação de fécula de mandioca e albedo de laranja. Alimentos e Nutrição, 21(3), 469-480.

Saunt, J. (2000). Citrus varieties of the word (2nd ed.). Norwich: Sinclair International Limite.

Souza, B. C. J., Costa, M. R., Derensis, C. M. V. B., \& Sivieri, K. (2010). Sorvete: Composição, processamento e viabilidade da adição de probiótico. Alimentos e Nutrição, 21(1), 155-165.

Stoll, L., Flores, S. H., \& Thys, R. C. S. (2015). Utilização de fibra de laranja como substituto de gordura em pão de forma. Ciência Rural, 24(3), 567-573. http://dx.doi.org/10.1590/0103-8478cr20131503

Talukder, S. (2015). Effect of dietary fiber on properties and acceptance of meat products: A review. Critical Reviews in Food Science and Nutrition, 55(7), 1005-1011. PMid:24915339. http://dx.doi.org/10.1080/10408398.2012.682230

Universidade Estadual de Campinas - UNICAMP. (2011). Tabela Brasileira de Composição de Alimentos: TACO (4. ed.). Campinas: UNICAMP/NEPA. 